\title{
List-method directed forgetting can be selective: Evidence from the 3-list and the 2-list tasks
}

\author{
Oliver Kliegl • Bernhard Pastötter • \\ Karl-Heinz T. Bäuml
}

Published online: 7 November 2012

(C) Psychonomic Society, Inc. 2012

\begin{abstract}
When people are cued to forget previously studied irrelevant information and study new information instead, such cuing typically leads to forgetting of the precue information. But what do people forget if, before the forget cue is provided, both irrelevant and relevant information have been encoded? Using relatively short item lists, we examined in a series of three experiments whether participants are able to selectively forget the irrelevant precue information, when relevant and irrelevant precue items were presented subsequently in two separate lists (3-list task) and when the two types of items were presented alternatingly within a single list (2-list task). Selective forgetting of the irrelevant precue items arose in the 3-list task, independent of modality of item presentation and level of discriminability of the precue lists, and it arose in the 2-list task. The findings suggest that, at least with relatively short precue lists, participants may well be able to selectively forget irrelevant precue information when cued to do so. Implications of the results for theoretical accounts of list-method directed forgetting are discussed.
\end{abstract}

\section{Keywords Episodic memory $\cdot$ Forgetting $\cdot$ Directed} forgetting $\cdot$ Selectivity

List-method directed forgetting (LMDF) is the demonstration that people can intentionally forget previously encoded information when cued to do so. In this paradigm, participants typically study two lists of items and, after study of list 1 , receive a cue either to forget or to continue remembering this list. The forget cue indicates that all of the precue information is irrelevant for an upcoming test and thus should be forgotten, whereas the remember cue indicates

O. Kliegl $\cdot$ B. Pastötter $\cdot$ K.-H. T. Bäuml $(\bowtie)$

Department of Experimental Psychology, Regensburg University, 93040 Regensburg, Germany

e-mail: karl-heinz.baeuml@psychologie.uni-regensburg.de that all of the precue information is relevant and thus should be remembered. After study of list 2, which is always to be remembered, participants are asked to recall the two lists' items irrespective of original cuing. The typical finding is that, as compared with remember-cued participants, forgetcued participants recall fewer list 1 items and more list 2 items, referred to as forgetting of the precue information and enhancement of the postcue information (for reviews, see Bäuml, Pastötter, \& Hanslmayr, 2010; MacLeod, 1998). ${ }^{1}$

LMDF has mostly been attributed to a single mechanism, regarded as responsible for both the forgetting of the precue information and the enhancement of the postcue information. The selective-rehearsal account, for instance, assumes that during postcue encoding, remember-cued participants rehearse both precue and postcue items, whereas forget-cued participants selectively rehearse postcue items, thus improving later recall of postcue items at the expense of the precue items (Bjork, 1970). The retrieval-inhibition account assumes that forget-cued participants engage in active inhibitory processes that impair access to the precue list, thus reducing recall of the precue items and, due to the resulting decrease in the precue items' interference potential, improving recall of the postcue items (Geiselman, Bjork, \& Fishman, 1983). Finally, the context-change account claims that the forget cue induces an internal context change that, at test, impairs recall of the precue items due to a mismatch between the context at encoding and the context at retrieval and, due to the reduced interference from the precue items, improves recall of the postcue items (Sahakyan \& Kelley, 2002). As an alternative to one-

\footnotetext{
${ }^{1}$ In the literature, two different directed-forgetting tasks have been employed: listwise and itemwise directed forgetting. In contrast to the listwise task, in the itemwise task, participants study a list of items, and the exposure of each single item is followed closely by the cue to either remember it or forget it. On a later memory task, remember-cued items are typically better recalled than forget-cued items (for reviews, see Bäuml, 2008; MacLeod, 1998; Pastötter \& Bäuml, 2010b).
} 
mechanism accounts of LMDF, two-mechanism accounts also have recently been suggested, assuming that the forgetting of the precue items is caused by inhibition or context change, whereas the enhancement of the postcue items (partly) reflects improved encoding (Pastötter, Kliegl, \& Bäuml, 2012; Sahakyan \& Delaney, 2003).

\section{Is LMDF selective?}

Recent work on LMDF has shown that cuing participants to forget a previously studied item list leads to forgetting of all the items on this list, impairing recall of the single items about equally (Pastötter \& Bäuml, 2010a; Pastötter et al., 2012; Sahakyan \& Foster, 2009). While such general forgetting of all the precue information appears adaptive if all the precue information has been designated as unimportant, such forgetting would be less advantageous if, before the forget cue was provided, both relevant and irrelevant information had been encoded and participants, in response to the forget cue, forgot all of the (relevant and irrelevant) precue information. For instance, when preparing for an exam, a student may receive information that some of the previously studied material is irrelevant for the upcoming test, whereas other parts of the material are likely to be tested. In such situations, LMDF would be adaptive only if the student was able to selectively forget the irrelevant precue information while keeping the relevant precue information in mind. Theoretical accounts of LMDF make different predictions as to whether people show selective forgetting or not.

The context-change account predicts that participants show no selectivity in LMDF. Because the account assumes that, in response to a forget cue, participants change internal context, an encoding-retrieval mismatch should arise for all previously studied items, regardless of whether they were all to be forgotten or consisted of a mixture of relevant and irrelevant items. In contrast, the selective-rehearsal account predicts that participants show a high level of selectivity in LMDF. Because this account claims that, after a forget cue is provided, (only) the irrelevant memories are skipped from the rehearsal process, participants should rehearse the relevant items regardless of whether they were presented before or after the forget cue was provided. The retrieval-inhibition account, in itself, makes no clear-cut prediction on participants' selectivity in LMDF. However, recent work relating performance in the LMDF task to individuals' working memory capacity (Aslan, Zellner, \& Bäuml, 2010; Delaney \& Sahakyan, 2007; Soriano $\&$ Bajo, 2007) and executive control function (Conway \& Fthenaki, 2003; Conway, Harries, Noyes, Racsmany, \& Frankish, 2000; Hanslmayr et al., 2012) suggests that retrieval inhibition may reflect the action of a fairly flexible control mechanism and, thus, may be targeted selectively at the irrelevant precue information.

\section{Prior work on selectivity in LMDF}

To our knowledge, there are only two published studies to date that have looked at selectivity in LMDF. Using a 3-list variant of the LMDF paradigm, Sahakyan (2004) presented relevant and irrelevant items subsequently in separate lists. Participants studied three item lists, each list consisting of either 12 unrelated words (Experiment 1) or 12 semantically related words from specific semantic categories (vegetables, fruits, and animals; Experiment 2). Each list was followed by a cue either to forget or to remember the preceding list. Participants were instructed to remember all three item lists (RRR), to forget list 1 but remember list 2 and list 3 (FRR), or to forget list 2 but remember list 1 and list 3 (RFR). At test, participants then were asked to recall the three lists' items irrespective of original cuing. As compared with the RRR condition, the results showed the expected effects of list 1 forgetting in the FRR condition and list 2 forgetting in the RFR condition, indicating effective listwise forgetting. More important, in both experiments, the results showed reliable list 1 forgetting in the RFR condition, indicating that the forgetting extended to the relevant precue information. The results are in line with the context-change account, which predicts no selectivity in LMDF, but are inconsistent with the selective-rehearsal account, which predicts a high level of selectivity in this paradigm. The results are also inconsistent with the retrievalinhibition account, at least if the inhibition is assumed to be mediated by an underlying flexible control mechanism.

A quite different picture on selectivity in LMDF arises from the results of a more recent study by Delaney, Nghiem, and Waldum (2009). Employing a 2-list variant of the LMDF paradigm, Delaney et al. examined participants' selectivity in LMDF by presenting relevant and irrelevant precue items as part of a common list. Participants studied two lists of items. List 1 consisted of 16 sentences. Half of the sentences were associated to the character of a putative person called Alex, and the other half were associated to the character of Tom. Alex and Tom sentences were presented in alternating order. For half of the participants, sentences were unrelated; for the other half, sentences of each of the two characters were semantically related. After study of list 1 , participants either were cued to remember Alex items but to forget Tom items or were cued to remember both Alex and Tom items. After study of list 2 , which consisted of 16 unrelated sentences associated to the third character of Joe, participants were asked to recall the items of all three characters, irrespective of original cuing. The results showed that, while there was no effect of cuing for semantically related items, cuing participants to selectively forget unrelated Tom items induced forgetting of Tom items without forgetting of Alex items, indicating that the forgetting did not extend to the not-irrelevant precue items - that is, was selective. The results are in line with both the selectiverehearsal account and the retrieval-inhibition account of LMDF but challenge the context-change account. 


\section{The present study}

Because the studies by Sahakyan (2004) and Delaney et al. (2009) differ in a number of factors, including differences in design, materials, and procedure, it is largely unclear why participants showed selective LMDF in the one study but not in the other. Apart from that, the results from the two studies may suggest that LMDF is not generally selective or nonselective but is present under some conditions and absent under others. In the present study, we examined selectivity of LMDF in the 3-list task under conditions that may favor selective forgetting. In contrast to Sahakyan's previous study, in which participants studied relatively long precue lists with 12 items each, in the present study participants studied relatively short precue lists with 6 items each. Short lists have sometimes been observed to induce very high levels of forgetting in the LMDF task (Conway \& Fthenaki, 2003; Conway et al., 2000); in particular, they may be easier to discriminate than long lists, which may enhance selective LMDF. Consistently, Delaney et al. used relatively short lists and found selective LMDF, whereas Sahakyan used relatively long lists and found nonselective LMDF.

While the main goal of the present study was to examine whether LMDF can be selective in the 3-list task when employing short precue lists, there were two additional issues that were addressed in this study. The first issue was an examination of whether selectivity in the 3-list task varies with the level of discriminability of the precue lists, assuming that with a high level of discriminability between the irrelevant precue list and the relevant precue list, selectivity may be high, and with a low level of discriminability, it may be low. Sahakyan (2004) addressed the issue by examining selectivity with lists of unrelated items in one experiment and with lists of semantically related items in the other, with largely identical results. The second issue was an examination of whether selectivity in LMDF depends on task and differs between the 3-list and the 2-list task. As was emphasized above, the studies by Sahakyan and by Delaney et al. (2009) differed in a number of ways, but they also differed in type of task, raising the possibility that selectivity in LMDF may be easier to achieve in the one (2-list) task than in the other (3-list) task.

In the following, the results of three experiments designed to address all of these issues are reported. In each of the three experiments, short precue sets of six items each were employed, with the aim to facilitate participants' selectivity in the task. Experiment 1 examined selective LMDF in the 3-list task. Discriminability of the relevant precue items (list 1) and the irrelevant precue items (list 2) was varied by presenting the items of the two lists either in the same font color or in different font colors. Experiment 2 also examined selective LMDF in the 3-list task. Again, discriminability of the relevant precue items (list 1) and the irrelevant precue items (list 2) was varied. This time the items were presented auditorily, by presenting the two lists either in the same voice or in different voices. Finally, Experiment 3 investigated whether selective LMDF varies between the 3-list task and the 2-list task. Holding both materials and other procedural detail constant, the experiment examined whether selectivity in LMDF is different if the irrelevant and the relevant precue items belong to separate lists and are presented successively (3-list task) than if the two types of items belong to the same list and are presented in an alternating order (2-list task).

\section{Experiment 1}

Experiment 1 examined selectivity in the 3-list task of LMDF by using visual item presentation and varying discriminability of the precue lists. Participants studied three visually presented lists of unrelated words. After study of list 2, participants were cued either to remember both list 2 and list 1 (RRR condition) or to forget list 2 but remember list 1 (RFR condition). Discriminability of the precue lists was manipulated by varying the items' font color. In the same-context condition, list 1 and list 2 items were presented in the same font color; in the different-context condition, they were presented in different font colors.

If participants in this task showed perfect selectivity, cuing them to selectively forget part of the precue information should induce the two typical LMDF effects - that is, forgetting of the irrelevant precue items (list 2) and enhancement of the relevant postcue items (list 3) - but should not induce forgetting of the relevant precue items (list 1). These results should be present regardless of context condition. Alternatively, if participants in this task were nonselective, again the two typical LMDF effects should arise - that is, forgetting of list 2 items and enhancement of list 3 items. In this case, however, forgetting of the relevant precue information (list 1) should be found as well. Again, all three effects should be present regardless of context condition. Finally, if participants' selectivity depended on context discriminability, the two typical LMDF effects should show up; however, list 1 forgetting should be present mainly in the high-discrimination condition but should be reduced, or even absent, in the low-discrimination condition.

Method

\section{Participants}

One hundred twenty-eight students (90 females and 38 males) at Regensburg University participated in Experiment 1. They were tested individually, with 32 participants in each of the four experimental conditions. 


\section{Materials}

Twenty-four unrelated German nouns of medium frequency were drawn from the CELEX database, using the Wordgen v1.0 software toolbox (Duyck, Desmet, Verbeke, \& Brysbaert, 2004). For each participant, three item lists were prepared. List 1 and list 2 consisted of 6 words; list 3 consisted of 12 words. For all participants, the assignment of items to lists was random.

\section{Design}

The experiment had a $2 \times 2$ design with the betweenparticipants factors of cuing (RRR, RFR) and context (same, different). Conditions differed in which cue was provided after list 2 . In the RRR condition, list 2 was followed by a cue to remember both list 2 and list 1; in the RFR condition, list 2 was followed by a cue to forget list 2 but remember list 1 . Conditions also differed in whether list 1 and list 2 items were presented in the same font color or in different font colors.

\section{Procedure}

The multiple-cue version of LMDF was used (see Pastötter \& Bäuml, 2007, 2010a; Zellner \& Bäuml, 2006). Participants were told that they would be presented with lists of words to learn for a later recall test and that, following each list, they would be given a cue to remember or forget the previously studied item list(s). Participants were told that an irrelevant list would not be tested on the later recall test. Prior to the encoding of each single list, participants were told that a "green" or a "blue" list of items would be presented next. The three lists" items were presented in the center of a computer monitor, with a presentation rate of $4 \mathrm{~s}$ per item. Item order within list was random for all participants. All words of a list were presented in either green or blue font color. In the same-context condition, list 1 and list 2 items were presented in the same font color; in the different-context condition, they were presented in different font colors. List 3 items were always presented in the same font color as list 2 items. List 1 encoding was always followed by a cue to remember this list. List 2 encoding was followed by a cue either to remember list 2 and keep on remembering list 1 (RRR) or to forget list 2 but keep on remembering list 1 (RFR). Following the encoding phase, participants counted backward from a three-digit number in steps of threes for $30 \mathrm{~s}$ as a recency control. At test, participants were asked to recall the three lists' items, irrespective of original cuing. Because the focus of this study was on precue item recall, participants were asked to recall precue lists first. Half of the participants recalled list 1 items first and list 2 items second; for the other half, list output order was reversed. All participants were asked to recall list 3 items last. Participants wrote down the items of the three lists on separate sheets of papers. Recall time for list 1 and list 2 items was $30 \mathrm{~s}$ each; recall time for list 3 items was $1 \mathrm{~min}$.

\section{Results}

Figure 1 shows mean recall rates as a function of cuing (RRR, RFR) and context (same, different), separately for the three lists. Items were counted as recalled if recalled with the correct list.

\section{List 1 recall}

A $2 \times 2$ analysis of variance (ANOVA) with the factors of cuing (RRR vs. RFR) and context (same vs. different) showed no main effect of cuing, $F(1,124)<1$, no main effect of context, $F(1,124)=1.32, M S E=.06, p=.252$, and
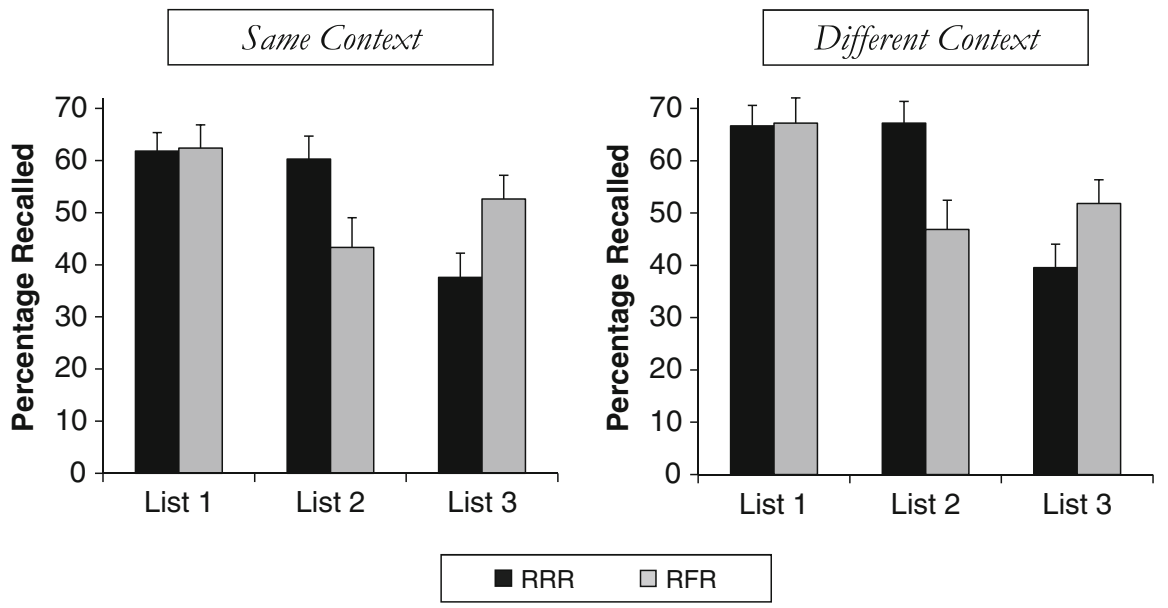

Fig. 1 Mean recall rates as a function of cuing (RRR, RFR) and context (same, different) in Experiment 1, separately for the three item lists. $\mathrm{RRR}=$ participants were asked to remember all three item lists; $\mathrm{RFR}=$ participants were asked to remember list 1 and list 3 but to

forget list 2 . Same context $=$ list 1 and list 2 items were presented in the same font color; different context $=$ list 1 and list 2 items were presented in different font colors. Error bars represent standard errors 
no interaction between factors, $F(1,124)<1$. Cuing participants to selectively forget list 2 thus did not induce list 1 forgetting, both when the precue lists were presented in the same context (61.9\% vs. $62.4 \%)$ and when they were presented in different contexts $(66.7 \%$ vs. $67.2 \%)$, ts $(62)<1$.

\section{List 2 recall}

A $2 \times 2$ ANOVA with the factors of cuing (RRR vs. RFR) and context (same vs. different) revealed a main effect of cuing, $F(1,124)=13.85, M S E=.08, p<.001$, partial $\eta^{2}=.10$, but no main effect of context, $F(1,124)=1.09, M S E=.08, p=.298$, and no interaction between the two factors, $F(1,124)<1$. Cuing participants to selectively forget list 2 thus induced list 2 forgetting, both when the precue lists were presented in the same context $(60.3 \%$ vs. $43.3 \%), t(62)=2.35, p=.022$, $d=.60$, and when they were presented in different contexts $(67.2 \%$ vs. $46.9 \%), t(62)=2.93, p=.005, d=.77$.

\section{List 3 recall}

A $2 \times 2$ ANOVA with the factors of cuing (RRR vs. RFR) and context (same vs. different) revealed a main effect of cuing, $F(1$, 124) $=9.00, M S E=.07, p=.003$, partial $\eta^{2}=.07$, but neither a main effect of context nor an interaction between the two factors, $F \mathrm{~s}(1,124)<1$. Cuing participants to selectively forget list 2 thus induced list 3 enhancement, both when the precue lists were presented in the same context $(37.6 \%$ vs. $52.6 \%), t(62)=1.93$, $p=.050, d=.48$, and when they were presented in different contexts $(39.6 \%$ vs. $51.8 \%), t(62)=2.32, p=.024, d=.59$.

\section{Intrusions}

Table 1 shows intrusion rates as a function of cuing (RRR vs. RFR) and context (same vs. different), separately for the three item lists. A list's intrusion rate represents the percentage of those items that were not recalled with the correct list but were erroneously recalled with one of the other lists. Intrusion rates were generally low, on the order of $3 \%$ in the single conditions. Three $2 \times 2$ ANOVAs with the factors of cuing (RRR vs. RFR) and context (same vs. different) showed no main effect or interaction, for all three item lists, all $F_{\mathrm{S}}<1.2$. Intrusions thus did not depend on cuing and list context.

\section{Discussion}

The results of Experiment 1 show the two typical LMDF effects, forgetting of the irrelevant precue items (list 2) and enhancement of the relevant postcue items (list 3 ). These effects were present in both the same-context and the different-context conditions. More important, the results of Experiment $1 \mathrm{dem}-$ onstrate selective LMDF; cuing participants to selectively forget list 2 did not induce list 1 forgetting, regardless of context condition. The results indicate that participants distinguished between relevant and irrelevant precue items and were able to forget the irrelevant items while keeping the relevant items in mind. The finding provides first evidence that selective LMDF is possible in the 3-list task, at least when short lists of items are employed that may be relatively easy to discriminate.

Concluding from the results of Experiment 1 that manipulating the level of discriminability of lists (same-context vs. different-context condition) does not play a major role for selectivity with short lists might be premature, however. In this experiment, the context manipulation did not affect overall recall rates, indicating that it may also not have affected discriminability of the precue lists. Indeed, if variation of font color had affected discriminability of the precue lists, overall recall rates in the different-context condition should have been higher than in the same-context condition, due to interference reduction. The issue was reexamined in Experiment 2, in which a different and possibly stronger manipulation of list context was employed.

\section{Experiment 2}

To our knowledge, Experiment 1 provides the first demonstration that LMDF in the 3-list task can be selective.

Table 1 Mean intrusion rates (and standard errors) as a function of cuing, context, and task in Experiments 1-3

\begin{tabular}{|c|c|c|c|c|c|c|c|}
\hline & & \multicolumn{2}{|c|}{ relevant precue items } & \multicolumn{2}{|c|}{ irrelevant precue items } & \multicolumn{2}{|l|}{ postcue items } \\
\hline & & same context & different context & same context & different context & same context & different context \\
\hline \multirow[t]{2}{*}{ Experiment 1} & $R R R$ & $2.1(1.3)$ & $3,6(1.4)$ & $1.6(0.9)$ & $3.1(1.4)$ & $2.5(0.8)$ & $1.8(0.7)$ \\
\hline & $R F R$ & $3.2(1.2)$ & $3,1(1.2)$ & $3.9(1.9)$ & $2.6(1.3)$ & $2.0(0.7)$ & $1.9(0.7)$ \\
\hline \multirow[t]{4}{*}{ Experiment 2} & $R R R$ & $5.7(2.3)$ & $5.2(2.0)$ & $2.1(1.0)$ & $4.7(2.1)$ & $3.9(1.5)$ & $4.7(2.2)$ \\
\hline & $R F R$ & $4.2(2.2)$ & $4.2(1.5)$ & $4.2(1.5)$ & $6.2(1.5)$ & $4.4(1.5)$ & $1.8(2.5)$ \\
\hline & $F F R$ & $4.7(1.7)$ & $4.7(1.7)$ & $6.7(2.5)$ & $5.9(1.9)$ & $1.6(0.6)$ & $2.4(0.9)$ \\
\hline & & 3-list task & 2-list task & 3-list task & 2-list task & 3-list task & 2-list task \\
\hline \multirow[t]{2}{*}{ Experiment 3} & remember & $4.0(1.0)$ & $5.0(0.9)$ & $7.6(1.6)$ & $4.4(0.9)$ & $3.2(0.6)$ & $2.2(0.6)$ \\
\hline & forget & $3.1(0.7)$ & $3.8(0.8)$ & $7.6(1.6)$ & $5.4(0.9)$ & $2.8(0.6)$ & $1.3(0.3)$ \\
\hline
\end{tabular}


The goal of Experiment 2 was to replicate the finding of Experiment 1 with different word material and different modality of item presentation. Participants studied three auditorily presented item lists. Discriminability of the precue lists was varied by presenting the two lists' items by either the same voice or two different voices. Experiment 2 employed the same two cuing conditions (RRR and RFR) as Experiment 1. However, a third cuing condition was included, in which study of list 2 was followed by a cue to forget both list 2 and list 1 (FFR). By including this condition, list 1 recall in the RFR condition could be compared with both an upper (RRR) and a lower (FFR) baseline condition, reflecting minimum (RRR) and maximum (FFR) forgetting of the relevant precue items.

We expected the results of Experiment 2 to replicate the selective LMDF finding of Experiment 1, showing list 2 forgetting without list 1 forgetting in the RFR condition. In particular, if the forgetting showed a high degree of selectivity, list 1 recall in the RFR condition should be the same as in the RRR condition but should be higher than in the FFR condition; in contrast, list 2 recall in the RFR condition should be the same as in the FFR condition but should be lower than in the RRR condition. Analysis of the effects of list context will show whether voice variation, in contrast to the items' font color in Experiment 1, affects discriminability of the precue lists and whether the variation in list discriminability influences participants' degree of selectivity.

\section{Method}

\section{Participants}

One hundred ninety-two students (129 females and 63 males) at Regensburg University participated in Experiment 2. They were tested individually, with 32 participants in each of the six experimental conditions.

\section{Materials}

Twenty-four unrelated German nouns of medium frequency were drawn from the CELEX database, which differed from the ones used in Experiment 1. For each participant, three item lists were prepared; the assignment of items to lists was random. List 1 and list 2 consisted of 6 words; list 3 consisted of 12 words. Prior to the experiment, for each of the 24 words, two auditory stimuli were created, consisting of 16-bit stereo speech from one female and one male adult speaker. Stimuli were recorded with a sampling rate of $22 \mathrm{kHz}$ and a maximum stimulus length of $1 \mathrm{~s}$. Recording and segmentation of auditory stimuli were done with Cool Edit 2000 v1.1 software.
Design

The experiment had a $3 \times 2$ design with the betweenparticipants factors of cuing (RRR, RFR, FFR) and context (same, different). Conditions differed as to which cue was provided after list 2 . In the RRR condition, list 2 was followed by a cue to remember both list 2 and list 1 ; in the RFR condition, list 2 was followed by a cue to forget list 2 but remember list 1 ; in the FFR condition, list 2 was followed by a cue to forget both list 2 and list 1 . Conditions also differed as to whether list 1 and list 2 items were presented by the same (male or female) voice or by different voices (a male voice and a female voice). In the same-context condition, list 1 and list 2 items were presented by the same voice; in the different-context condition, list 1 and list 2 items were presented by different voices.

\section{Procedure}

The procedure was identical to that in Experiment 1, with the only exception that the three lists' items were presented auditorily with a presentation rate of $4 \mathrm{~s}$ through standard headphones by either a female or a male voice. List 3 items were always presented by the same voice as list 2 items.

\section{Results}

Figure 2 shows mean recall rates as a function of cuing (RRR, RFR, FFR) and context (same, different), separately for the three lists. Items were counted as recalled if recalled with the correct list.

\section{List 1 recall}

A $3 \times 2$ ANOVA with the factors of cuing (RRR vs. RFR vs. FFR) and context (same vs. different) showed a main effect of cuing, $F(2,186)=11.70, M S E=.07, p<.001$, partial $\eta^{2}=.11$, and a main effect of context, $F(1,186)=21.51, M S E=.07$, $p<.001$, partial $\eta^{2}=.10$, but no interaction between factors, $F(2,186)<1$. List 1 recall rates were higher when precue lists were presented in different contexts than when they were presented in the same context ( $60.1 \%$ vs. $43.2 \%)$. Regarding the main effect of cuing, pairwise comparisons showed that list 1 recall rates in the FFR condition (38.5\%) were lower than in both the RRR condition (58.1\%), $t(126)=4.00, p<.001$, $d=.73$, and the RFR condition $(57.8 \%), t(126)=3.72$, $p<.001, d=.65$, whereas list 1 recall rates did not differ between the RFR and RRR conditions, $t(126)<1$. These results indicate that list 1 forgetting was present in the FFR condition but was absent in the RFR condition, both when the precue lists were presented in the same context and when they were presented in different contexts. 


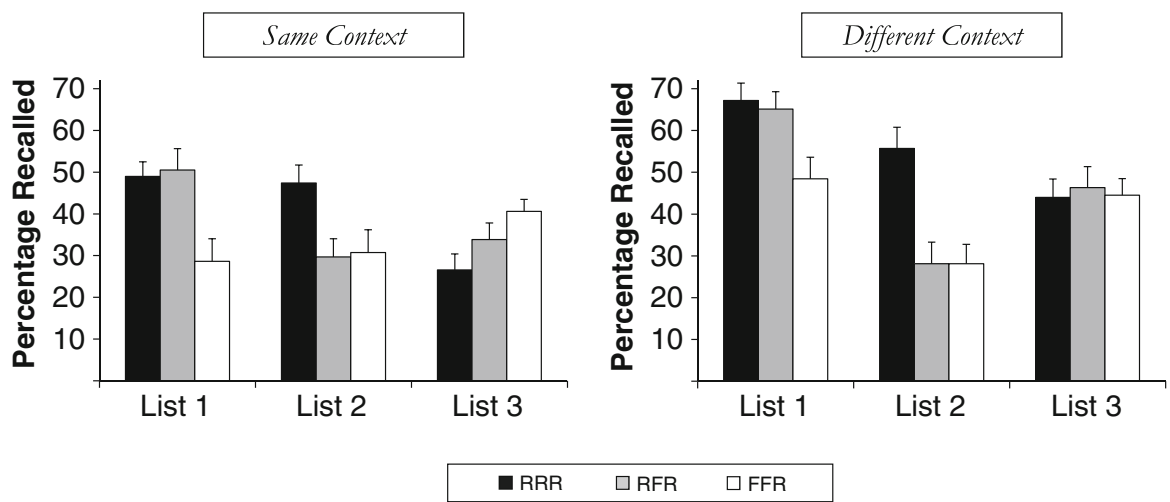

Fig. 2 Mean recall rates as a function of cuing (RRR, RFR, FFR) and context (same, different) in Experiment 2, separately for the three item lists. $\mathrm{RRR}=$ participants were asked to remember all three item lists; $\mathrm{RFR}=$ participants were asked to remember list 1 and list 3 but to

\section{List 2 recall}

A $3 \times 2$ ANOVA with the factors of cuing (RRR vs. RFR vs. FFR) and context (same vs. different) revealed a main effect of cuing, $F(2,186)=14.25, M S E=.08, p<.001$, partial $\eta^{2}=.13$, but neither a main effect of context, $F(1,186)<1$, nor an interaction between factors, $F(2,186)<1$. Pairwise comparisons showed that, as compared with the RRR condition $(51.6 \%)$, list 2 recall rates were lower in both the FFR condition $(29.4 \%), t(126)=4.54, p<.001, d=.81$, and the RFR condition $(28.9 \%), t(126)=4.80, p<.001, d=.88$; list 2 recall rates did not differ between the FFR and RFR conditions, $t(126)<1$. These results indicate that list 2 forgetting was present in both the FFR condition and the RFR condition, both when the precue lists were presented in the same context and when they were presented in different contexts.

\section{List 3 recall}

A $3 \times 2$ ANOVA with the factors of cuing (RRR vs. RFR vs. FFR) and context (same vs. different) revealed a main effect of context, $F(1,186)=11.67, M S E=.05, p<.001$, partial $\eta^{2}=$ .06 , but neither a main effect of cuing, $F(2,186)=1.68, M S E=$ $.05, p=.190$, nor an interaction between factors, $F(2,186)=$ $1.68, M S E=.05, p=.240$. List 3 recall rates were higher when precue lists were presented in different contexts than when they were presented in the same context (45.0\% vs. $33.7 \%$ ). No reliable list 3 enhancement was observed.

\section{Intrusions}

Table 1 shows intrusion rates in Experiment 2, separately for the three item lists. Three $3 \times 2$ ANOVAs with the factors of cuing (RRR vs. RFR vs. FFR) and context (same vs. different) showed no main effects or interaction, for all three item lists, all forget list 2; FFR $=$ participants were asked to forget both list 1 and list 2 but to remember list 3 . Same context $=$ list 1 and list 2 items were presented by the same voice; different context $=$ list 1 and list 2 items were presented by different voices. Error bars represent standard errors

$F_{\mathrm{S}}<1.8$. Intrusion rates were generally low, on the order of $4 \%$ in the single conditions, independently of cuing and list context.

\section{Discussion}

The results of Experiment 2 show typical LMDF of the irrelevant precue items. In the FFR condition, cuing participants to forget both precue lists induced forgetting of both list 1 and list 2; similarly, cuing participants to forget list 2 in the RFR condition induced list 2 forgetting. These effects were present in both the same-context and the different-context conditions. More important, the results again demonstrate selectivity in LMDF. In the RFR condition, cuing participants to forget list 2 but not list 1 did not induce list 1 forgetting, regardless of context condition. The findings agree with the results of Experiment 1, suggesting a high level of selectivity in LMDF with short precue lists. In addition, the finding that context affected overall recall rates but did not affect selectivity supports the view that, under the conditions employed, selectivity in LMDF is possible even with a relatively low degree of list discriminability.

The finding of no enhancement of the list 3 postcue items disagrees with the results of Experiment 1 and also disagrees with the results of many studies in the LMDF literature. On the other hand, the finding is in line with results from more recent LMDF work (e.g., Delaney \& Sahakyan, 2007; Delaney et al., 2009; Sahakyan, 2004; Zellner \& Bäuml, 2006), which shows that the enhancement is not always present in LMDF. Pastötter et al. (2012) recently demonstrated that the failure to find the enhancement effect often occurs when, at test, the precue items are recalled first and the postcue items are recalled last, a procedure that was also chosen in the present experiment. The General Discussion section below provides a theoretical explanation of this output order effect and discusses the issue further.

Lehman and Malmberg (2009) recently suggested a modification of the standard LMDF task, arguing that the task may 
lead to a confounding of list number with the presence versus absence of a prior list, because only list 1, but not the more recent list(s), is not preceded by another list. For instance, the present finding of equivalent list 1 recall in the RFR and RRR conditions might, in part, be due to the fact that list 1 was not preceded by another list and participants may have easily reinstated the list's original study context, thus showing perfect selectivity. First, in the present study, we did not follow the Lehman-Malmberg suggestion in order to stay comparable in methods with the previous studies on selectivity in LMDF (Delaney et al., 2009; Sahakyan, 2004). Second, it seems quite unlikely that participants easily reinstated the original (list 1) study context in the present experiments, because lists 1 and 2 showed reliable and equivalent forgetting in the current FFR conditions. Such pattern should not have emerged if context reinstatement for list 1 was easy or if reinstatement was at least more easy for list 1 , which was not preceded by another list, than for list 2, which was preceded by another list. The finding therefore challenges the view that the absence or presence of a prior list constituted a major confounding in the present experiments.

\section{Experiment 3}

In the prior work on selectivity in LMDF, Sahakyan (2004) did not find evidence for selectivity when using the 3-list task, whereas Delaney et al. (2009) reported such evidence when using the 2-list task. Although the two studies differed not only in task, but also in other aspects, including materials and procedural detail (see the introduction), one possibility for the conflicting results might be that selectivity in LMDF varies with task and is easier to achieve in the one (2-list) task than in the other (3-list) task. To date, this issue has not been investigated. The goal of Experiment 3 was to provide such a comparison and to examine directly whether selectivity in LMDF varies with type of task.

In both the 3-list and 2-list tasks of Experiment 3, relevant and irrelevant precue items were spoken by two different speaker voices, identical to the different-context condition of Experiment 2. While in the 3-list task, relevant and irrelevant items were again assigned to separate lists and were presented successively as lists 1 and 2, in the 2-list task, relevant and irrelevant precue items were assigned to the same list and were presented in an alternating order. In both tasks, after study of these items, participants were cued to forget the irrelevant precue items but to keep the relevant precue items in mind ([selective] forget condition), or to remember all precue items (remember condition). In both tasks, perfect selectivity would be reflected in decreased recall rates for the irrelevant precue items in the forget condition, relative to the remember condition, and similar recall levels for the relevant precue items in the remember and forget conditions. On the basis of the results of
Experiments 1 and 2, we expected selectivity in the 3-list task. On the basis of the results by Delaney et al. (2009), we expected a similar, or even higher, level of selectivity in the 2-list task.

Method

\section{Participants}

Two hundred forty students (139 females, 101 males) at Regensburg University participated in Experiment 3. All participants were tested individually, with 120 participants in each of the two tasks.

\section{Materials}

Forty-eight unrelated German nouns of medium frequency were drawn from the CELEX database, which differed from those in Experiments 1 and 2. For each participant, six item sets were prepared: 3 sets for the forget condition and 3 sets for the remember condition. The assignment of items to sets was random. Sets 1 and 2 in each condition consisted of 6 items each, set 3 of 12 items. In the 3 -list task, sets 1, 2, and 3 were presented as lists 1,2, and 3; in the 2-list task, sets 1 and 2 were presented as list 1 , and set 3 was presented as list 2 . For each of the 48 items, two auditory stimuli were created, consisting of 16-bit stereo speech from one female and one male speaker. Recording and segmentation were conducted as in Experiment 2.

\section{Design}

The experiment had a $2 \times 2$ mixed design with the withinparticipants factor of cuing (forget vs. remember) and the between-participants factor of task (3-list task, 2-list task). Conditions differed as to whether the relevant and irrelevant precue items were assigned to separate lists and were presented blocked (3-list task) or the two sets of items were part of the same list and were presented in an alternating order (2-list task). Conditions also differed as to what type of cue was provided, either a cue to forget half of the precue items but to keep the remaining precue items in mind (forget condition) or a cue to remember all precue items (remember condition).

\section{Procedure}

In the 3-list task, the procedure was identical to the differentcontext condition of Experiment 2, with the only exception that each participant took part in both cuing conditions. The 2-list task was identical to the 3-list task, with the only difference that the relevant and irrelevant precue items were presented alternatingly as part of the same list (list 1) and the remember or forget cue was presented after study of the list. 
Analogously to the 3-list task, in the 2-list task participants at test were asked either to recall the irrelevant precue items first and the relevant precue items second or vice versa. The postcue items were always tested last. Testing details were identical to those for the 3-list task. Between the two experimental conditions, there was a break of $30 \mathrm{~s}$ before the next condition started.

Results

Figure 3 shows mean recall rates as a function of cuing (forget, remember) and task (3-list task, 2-list task), separately for each of the three item types. ${ }^{2}$

\section{Recall of relevant precue items}

A $2 \times 2$ ANOVA with the factors of cuing (forget vs. remember) and task (3-list task vs. 2-list task) revealed a main effect of cuing, $F(1,238)=7.76, M S E=.04, p=.006$, $\eta_{\mathrm{p}}^{2}=.03$, and a main effect of task, $F(1,238)=23.05, M S E=$ $.13, p<.001, \eta_{\mathrm{p}}^{2}=.09$, but no interaction between factors, $F$ $(1,238)=1.01, M S E=.04, p=.317$. Recall rates of relevant precue items were higher in the 3-list task than in the 2-list task ( $61.3 \%$ vs. $45.6 \%)$, and they were higher in the forget condition than in the remember condition $(56.0 \%$ vs. $50.8 \%)$. Pairwise comparisons showed that the effect of condition was reliable in the 2 -list task $(49.2 \%$ vs. $42.1 \%), t(119)=3.15$, $p=.002, d=.41$, but was not reliable in the 3 -list task $(62.9 \%$ vs. $59.6 \%), t(119)=1.12, p=.267$. These results indicate that, in both tasks, there was no cue-induced forgetting of relevant precue items; rather, there was (a tendency for) a small beneficial effect of the forget cue.

\section{Recall of irrelevant precue items}

A $2 \times 2$ ANOVA with the factors of cuing (forget vs. remember) and task (3-list task vs. 2-list task) revealed a main effect of cuing, $F(1,238)=22.69, M S E=.05, p<.001, \eta_{\mathrm{p}}^{2}=.09$, and a main effect of task, $F(1,238)=10.96, M S E=.11, p<.001$, $\eta_{\mathrm{p}}^{2}=.04$, but no interaction between factors, $F(1,238)=1.30$, $M S E=.05, p=.255$. Recall of irrelevant precue items was higher in the 3 -list task than in the 2 -list task $(47.5 \%$ vs. $37.4 \%$ ), and it was lower in the forget condition than in the remember condition ( $37.5 \%$ vs. $47.4 \%$ ). Pairwise comparisons

\footnotetext{
${ }^{2}$ In Experiment 3, we chose the within-participants design of LMDF, with each participant participating in both a remember and a forget condition. Prior behavioral work reported no effect of order of remember and forget conditions on participants' LMDF (e.g., Bäuml \& Samenieh, 2012; Conway \& Fthenaki, 2003; Zellner \& Bäuml, 2006), thus providing an empirical rationale for our choice of design. Consistent with this prior work, order of cue conditions also did not affect the results of Experiment3 (all $p \mathrm{~s}>.05$ ).
}

showed that the effect of condition was reliable in both the 3 -list task $(41.4 \%$ vs. $53.6 \%), t(119)=3.75, p<.001$, $d=.49$, and the 2 -list task $(33.6 \%$ vs. $41.1 \%), t(119)=2.94$, $p=.004, d=.38$.

\section{Recall of postcue items}

A $2 \times 2$ ANOVA with the factors of cuing (forget vs. remember) and task (3-list task vs. 2-list task) showed no main effect of cuing, $F(1,238)<1$, no main effect of task, $F$ $(1,238)=1.43, M S E=.11, p=.234$, and no interaction between factors, $F(1,238)<1$. Cuing participants to selectively forget some of the precue items thus did not induce enhancement of the postcue items, neither in the 3-list task $(40.83 \%$ vs. $41.18 \%), t(119)<1$, nor in the 2 -list task (45.3\% vs. $44.1 \%), t(119)<1$.

\section{Intrusions}

Table 1 shows intrusion rates in Experiment 3, separately for each set. Three $2 \times 2$ ANOVAs with the factors of cuing (forget vs. remember) and task (3-list task vs. 2-list task) showed no main effects or interaction, for relevant precue items, for irrelevant precue items, or for postcue items. all $F_{\mathrm{s}}<2.3$. Intrusion rates were generally low, on the order of $4 \%$ in the single conditions, independently of cuing and task.

\section{Discussion}

The results in the 3-list task replicate the results of Experiments 1 and 2. They show typical LMDF of the irrelevant precue items, and they show selective forgetting of these items. Indeed, cuing participants to selectively forget the List 2 items caused forgetting of these items, but it did not cause forgetting of the relevant List 1 items. As in Experiment 2, there was no enhancement effect for the postcue items. The results support the view that selective LMDF is possible in the 3-list task.

The results in the 2-list task also show typical LMDF of the irrelevant precue items, and they also show selective forgetting of these items. Cuing participants to selectively forget half of the previously studied items caused forgetting of these items, but it did not cause forgetting of the remaining list items. Rather, there was even a small beneficial effect for these precue items, which may reflect a liststrength or list-length effect for the relevant items, assuming that access to the competing irrelevant items was reduced or even (partly) eliminated by means of the forget cue. As in the 3-list task, there was no enhancement effect for the postcue items. All of these findings replicate the results reported in Delaney et al. (2009) and, thus, confirm the view that selective LMDF is possible in the 2-list task. 

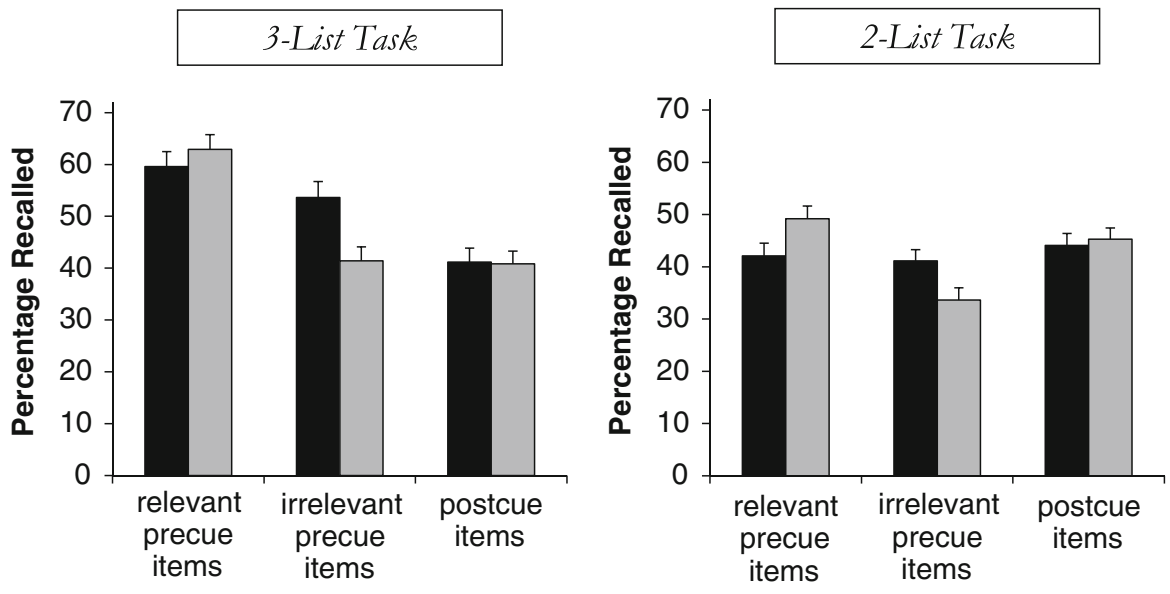

a remember $\square$ forget

Fig. 3 Mean recall rates as a function of cuing (forget vs. remember), and task (3-list task, 2-list task) in Experiment 3, separately for relevant precue items, irrelevant precue items, and postcue items. Remember $=$ participants were asked to remember all precue items as well as the

The direct comparison of results between the 3-list and the 2-list task did not provide any evidence for a difference in LMDF between the two types of tasks. The two LMDF tasks did not differ in forgetting of irrelevant precue items, "forgetting" of relevant precue items, and enhancement of the postcue items. The only difference between tasks was a difference in general recall level, with higher recall rates in the 3-list than in the 2-list task. This difference may reflect the well-known finding that recall of a set of items is typically better when the items are presented as two separate, shorter lists than when being part of a single larger list. The conflict in results between the studies of Sahakyan (2004) and Delaney et al. (2009) thus should not be due to the difference in task-3-list versus 2-list task-but should be caused by other factors (see below).

\section{General discussion}

Across three experiments, this study examined selectivity in LMDF, employing relatively short precue item sets that were supposed to enhance discriminability of item sets and, thus, to facilitate selectivity in LMDF. In all three experiments, evidence for selectivity arose. When cued to do so, participants were well able to selectively forget irrelevant precue material without any forgetting of the relevant precue items. Experiments 1 and 2 showed such selectivity in the 3-list task, with different modalities of item presentation and different levels of list discriminability; Experiment 3 reported such selectivity in both the 3-list task and the 2-list task, with equivalent levels of selectivity between tasks. postcue items; forget $=$ participants were asked to forget the irrelevant precue items but to remember the relevant precue items as well as the postcue items. Error bars represent standard errors

\section{Relation to prior work on selectivity in LMDF}

This is the first study in the literature showing that selective LMDF is possible in the 3-list task of LMDF. The finding, of course, contrasts with the results reported in Sahakyan (2004), in which no evidence for selectivity was found in two experiments. While Experiment 1 in Sahakyan's study is similar to the same-context conditions of the present Experiments 1 and 2 and Experiment 2 in Sahakyan's study is similar to the differentcontext conditions of the present Experiments 1-3-although in Sahakyan's experiment, precue lists differed in semantic category rather than font color or speaker's voice - there is also a number of methodological differences between the two studies that may account for the difference in results (see the introduction). The most striking methodological difference between studies, however, may be the length of the precue item lists, with the present study employing relatively short precue lists and the prior work employing relatively long precue lists. Length of precue lists may affect discriminability of the lists and, thus, affect selectivity in LMDF.

At first glance, the proposal that list discriminability affects selectivity in LMDF may seem in conflict with the finding of Experiments 1 and 2 that selectivity does not vary with list context of the precue lists. However, selectivity in the samecontext conditions of Experiments 1 and 2 was already at ceiling, thus leaving no room for increased selectivity in the different-context conditions. Arguably, for short precue lists, list discrimination may be sufficiently easy so that a high level of selectivity may arise even if the lists are presented in similar context. In contrast, for longer precue lists, discrimination may be more demanding, so that level of selectivity may be low when the lists do not differ in context and be enhanced, or even 
perfect, when the lists differ in context. Future work may like to address this hypothesis by examining whether, for longer lists, selectivity in LMDF varies as a function of context condition. ${ }^{3}$

This study is the second study in the literature showing that selective LMDF is possible in the 2-list task of LMDF. Indeed, the results of Experiment 3 show a reliable selectivity effect that is similar in size to the one reported in Delaney et al. (2009). Because Delaney et al. used materials that were quite different from the ones used in the present study (see the introduction), the finding suggests that materials may not play a major role for selective LMDF and selectivity may be possible both with simple word lists and more complex item materials. In particular, like the present study, Delaney et al. used relatively short precue item sets. The consistency in results between studies thus supports the view that selectivity is possible with relatively short precue item sets.

The conflicting results from prior work on selectivity in LMDF (Delaney et al., 2009; Sahakyan, 2004) raised the possibility that selectivity in LMDF may depend on task and be different in the 3-list task than in the 2-list task. The results from the present Experiment 3 suggest otherwise, however. Holding material and other procedural aspects constant, largely identical results arose in the two LMDF tasks, with regard to participants' recall pattern. The parallel indicates that task is not a major factor for selectivity in LMDF, and results from one task may well generalize to the other task. Such parallel is practically useful, because future studies can focus on one of the two tasks to study selectivity in LMDF.

\section{Theoretical implications for LMDF accounts}

The present results impose restrictions on theoretical accounts of LMDF. The forgetting of irrelevant precue items has often been explained by selective rehearsal, retrieval inhibition, or context change. The results of this study are in line with two of these accounts but challenge the third. The context-change account attributes the forgetting of irrelevant precue items to a change in internal context after precue item encoding, which, for the precue items, causes a mismatch between the context at encoding and the context at retrieval (Sahakyan \& Kelley, 2002). On the basis of this account, LMDF should not be selective, in both the 3-list and the 2-list task, because, if participants changed internal context after study of the relevant and irrelevant precue items, this should lead to an

\footnotetext{
${ }^{3}$ The suggestion seems to be in conflict with the findings of Sahakyan's (2004) previous study, which employed longer lists, also varied list discriminability, but reported no effect of list discriminability on selective LMDF. In this previous study, however, list discriminability was manipulated across experiments, and the single experiments not only differed in list discriminability, but also differed in a number of further methodological aspects, such as, for instance, control of list output order. Concluding from this prior work that, with longer precue lists, selectivity does not depend on list discriminability thus might be premature.
}

encoding-retrieval mismatch for both types of items. The present finding that LMDF is restricted to the irrelevant precue items disagrees with this prediction and, thus, challenges the context-change account.

In contrast, the present results are consistent with the selective-rehearsal account (Bjork, 1970), which predicts selective LMDF. This account assumes that in the selective forget condition, only the irrelevant memories are skipped from participants' rehearsal process, creating forgetting of irrelevant precue items without forgetting of relevant precue items. While the retrieval-inhibition account (Geiselman et al., 1983) in itself makes no unequivocal prediction about selectivity in LMDF, following the view that retrieval inhibition is a fairly flexible control mechanism (e.g., Aslan et al., 2010; Conway et al., 2000; Hanslmayr et al., 2012), the retrieval-inhibition account suggests that participants in the selective forget condition may well be able to target inhibition to the irrelevant precue items without affecting the relevant precue material. The present findings agree with the predictions of both accounts and suggest both selective rehearsal and (a version of) retrieval inhibition as possible explanations of the selectivity finding.

While the present results thus favor the selective-rehearsal and retrieval-inhibition accounts of LMDF over the contextchange account, they, of course, do not imply that selective rehearsal or inhibition necessarily mediates LMDF in all types of situations. Rather, a more realistic proposal may be that LMDF can be mediated by different mechanisms in different situations and that even different individuals can employ different strategies for successful LMDF (for a similar point made in the think/no-think literature, see Levy \& Anderson, 2008). On the basis of such a view, the present results suggest that while selective rehearsal and inhibition may be viable LMDF mechanisms in most empirical situations, successful LMDF via context change may be limited to tasks in which the relevancy of items is confounded with list number and the precue list is designated as unimportant and the postcue list as important for an upcoming memory test.

\section{Postcue enhancement in LMDF}

While reliable (selective) forgetting of the (irrelevant) precue items was present in all three experiments in this study, reliable enhancement of the postcue items was observed only in Experiment 1. Neither in Experiment 2 nor in Experiment 3 did a beneficial effect of cuing for postcue items arise. This is not an unusual finding. There are a number of studies in the LMDF literature, including those on selectivity in LMDF (e.g., Delaney \& Sahakyan, 2007; Delaney et al., 2009; Pastötter \& Bäuml, 2010a; Sahakyan, 2004; Spillers \& Unsworth, 2011; Zellner \& Bäuml, 2006), showing that forgetting of the precue information can arise without enhancement of the postcue information. The dissociation has even been taken as support for the view that forgetting and enhancement in LMDF may be mediated 
by different mechanisms (Pastötter \& Bäuml, 2010a; Sahakyan \& Delaney, 2003; see also Bäuml, Hanslmayr, Pastötter, \& Klimesch, 2008; Hanslmayr et al., 2012).

Quite often, the failure to observe enhancement of the postcue items occurs when, at test, the precue items are recalled first and the postcue items are recalled last. This has recently been documented in a meta-analysis and has been shown experimentally by varying list output order at test (Pastötter et al., 2012). Enhancement of the postcue items in response to the forget cue has often been attributed to reduced interference of the precue items at test (e.g., Geiselman et al., 1983; Sahakyan \& Kelley, 2002). Because recall of some of a list's irrelevant items can improve recall of the list's remaining items (Bäuml \& Samenieh, 2010, 2012), prior testing of the precue items may release the precue items' interference potential and, thus, reduce subsequent enhancement of the postcue items. Such release may have happened in the present Experiments 2 and 3, as well as in the studies of Delaney et al. (2009) and Sahakyan (2004, Experiment 1), because in all these experiments, precue items were tested first at test and postcue items were tested last. Such release may even have occurred in Experiment 1, although obviously a reliable enhancement effect remained. Thus, to examine the effects of selective forgetting of precue information on the enhancement of the postcue information, fresh experiments are necessary in which the postcue items are recalled first at test, rather than last.

\section{Conclusions}

The results from the present series of experiments demonstrate that forgetting in the LMDF task can be selective, at least when relatively short precue sets of items have been studied. Under such conditions, after study of both relevant and irrelevant precue information, participants appear well able to selectively forget the irrelevant material while keeping the relevant items in mind. The selectivity finding was robust and was not affected by modality of item presentation, the level of discriminability of precue lists, and type of the LMDF task. Practically, the finding suggests that LMDF can be a valuable cognitive technique when selective forgetting is eligible. Theoretically, it is consistent with both the selective-rehearsal and the retrieval-inhibition accounts of LMDF but challenges the context-change account.

Authors' note This work was supported by a grant from the German Research Foundation (Deutsche Forschungsgemeinschaft, DFG) awarded to K.-H. T. B. and B. P. (BA 1382/11-1). The authors thank M. Brückl, S. Kaltner, A. Karl, M. Sieber, and M. Wiedemann for their help with data collection.

Correspondence concerning this article should be addressed to Karl-Heinz T. Bäuml, Department of Experimental Psychology, Regensburg University, 93040 Regensburg, Germany. E-mail: karl-heinz.baeuml@psychologie.uniregensburg.de.

\section{References}

Aslan, A., Zellner, M., \& Bäuml, K.-H. T. (2010). Working memory capacity predicts listwise directed forgetting in adults and children. Memory, 18, 442-450.

Bäuml, K.-H. (2008). Inhibitory processes. In H. L. Roediger III (Ed.), Cognitive psychology of memory. Vol. 2 of Learning and memory - a comprehensive reference (pp. 195-220). Oxford: Elsevier.

Bäuml, K.-H., Hanslmayr, S., Pastötter, B., \& Klimesch, W. (2008). Oscillatory correlates of intentional updating in episodic memory. NeuroImage, 41, 596-604.

Bäuml, K.-H., Pastötter, B., \& Hanslmayr, S. (2010). Binding and inhibition in episodic memory - Cognitive, emotional, and neural processes. Neuroscience and Biobehavioral Reviews, 34, 1047-1054.

Bäuml, K.-H. T., \& Samenieh, A. (2010). The two faces of memory retrieval. Psychological Science, 21, 793-795.

Bäuml, K.-H. T., \& Samenieh, A. (2012). Selective memory retrieval can impair and improve retrieval of other memories. Journal of Experimental Psychology: Learning, Memory, and Cognition, 38, 488-494.

Bjork, R. A. (1970). Positive forgetting: The noninterference of items intentionally forgotten. Journal of Verbal Learning and Verbal Behavior, 9, 255-268.

Conway, M. A., \& Fthenaki, A. (2003). Disruption of inhibitory control of memory following lesions to the frontal and temporal lobes. Cortex, 39, 667-686.

Conway, M. A., Harries, K., Noyes, J., Racsmany, M., \& Frankish, C. R. (2000). The disruption and dissolution of directed forgetting: Inhibitory control of memory. Journal of Memory and Language, 43, 409-430.

Delaney, P. F., Nghiem, K. N., \& Waldum, E. R. (2009). The selective directed forgetting effect: Can people forget only part of a text? The Quarterly Journal of Experimental Psychology, 62, 1542-1550.

Delaney, P. F., \& Sahakyan, L. (2007). Unexpected costs of high working memory capacity following directed forgetting and contextual change manipulations. Memory \& Cognition, 35, 1074 1082.

Duyck, W., Desmet, T., Verbeke, L., \& Brysbaert, M. (2004). Wordgen: A tool for word selection and non-word generation in Dutch, German, English, and French. Behavior Research Methods, Instruments, \& Computers, 36, 488-499.

Geiselman, R. E., Bjork, R. A., \& Fishman, D. (1983). Disrupted retrieval in directed forgetting: A link with posthypnotic amnesia. Journal of Experimental Psychology. General, 112, 58-72.

Hanslmayr, S., Volberg, G., Wimber, M., Oehler, N., Staudigl, T., Hartmann, T., Raabe, M., Greenlee, M. W., \& Bäuml, K.-H. T. (2012). Prefrontally driven down-regulation of neural synchrony mediates goal-directed forgetting. The Journal of Neuroscience, $32,14742-14751$.

Lehman, M., \& Malmberg, K. J. (2009). A global theory of remembering and forgetting from multiple lists. Journal of Experimental Psychology: Learning, Memory, and Cognition, $35,970-988$.

Levy, B. J., \& Anderson, M. C. (2008). Individual differences in the suppression of unwanted memories: The executive deficit hypothesis. Acta Psychologica, 127, 623-635.

MacLeod, C. M. (1998). Directed forgetting. In J. M. Golding \& C. M. MacLeod (Eds.), Intentional forgetting: Interdisciplinary approaches (pp. 1-57). Mahwah, NJ: Erlbaum.

Pastötter, B., \& Bäuml, K.-H. (2007). The crucial role of postcue encoding in directed forgetting and context-dependent forgetting. Journal of Experimental Psychology: Learning, Memory, and Cognition, 33, 977-982. 
Pastötter, B., \& Bäuml, K.-H. (2010a). Amount of postcue encoding predicts amount of directed forgetting. Journal of Experimental Psychology: Learning, Memory, and Cognition, 36, 54-65.

Pastötter, B., \& Bäuml, K.-H. T. (2010b). Retrieval inhibition in autobiographical memory. In J. H. Mace (Ed.), The act of remembering: Toward an understanding of how we recall the past (pp. 202-227). Wiley: Blackwell.

Pastötter, B., Kliegl, O., \& Bäuml, K.-H. T. (2012). List-method directed forgetting: the forget cue improves both encoding and retrieval of postcue information. Memory \& Cognition, 40, 861-873.

Sahakyan, L. (2004). Destructive effects of "forget" instructions. Psychonomic Bulletin \& Review, 11, 555-559.

Sahakyan, L., \& Delaney, P. F. (2003). Can encoding differences explain the benefits of directed forgetting in the list method paradigm? Journal of Memory and Language, 48, 195-206.
Sahakyan, L., \& Foster, N. L. (2009). Intentional forgetting of actions: Comparison of list-method and item-method directed forgetting. Journal of Memory and Language, 61, 134-152.

Sahakyan, L., \& Kelley, C. M. (2002). A contextual change account of the directed forgetting effect. Journal of Experimental Psychology: Learning, Memory, and Cognition, 28, 1064-1072.

Soriano, M. F., \& Bajo, M. T. (2007). Working memory resources and interference in directed forgetting. Psicológica, 28, 63-85.

Spillers, G. J., \& Unsworth, N. (2011). Are the costs of directed forgetting due to failures of sampling or recovery? Exploring the dynamics of recall in list-method directed forgetting. Memory \& Cognition, 39, 403-411.

Zellner, M., \& Bäuml, K.-H. (2006). Inhibitory deficits in older adults list-method directed forgetting revisited. Journal of Experimental Psychology: Learning, Memory, and Cognition, 32, 290-300. 